\title{
Modal Decomposition of the Global Response to Tropical Heating Perturbations Resembling MJO
}

\author{
KATARINA KOSOVELJ \\ University of Ljubljana, Ljubljana, Slovenia \\ FRED KUCHARSKI \\ ICTP, Trieste, Italy \\ FrANCO MOLTENi \\ ECMWF, Reading, United Kingdom \\ NEDJELJKA ŽAGAR ${ }^{\mathrm{a}}$ \\ University of Ljubljana, Ljubljana, Slovenia
}

(Manuscript received 17 July 2018, in final form 24 February 2019)

\begin{abstract}
The paper presents four ensembles of numerical experiments that compare the response to monopole and dipole heating perturbations resembling different phases of the Madden-Julian oscillation (MJO). The results quantify the Rossby and inertio-gravity (IG) wave response using the normal-mode function decomposition. The day 3 response is characterized by about $60 \%$ variance in the IG modes, with about $85 \%$ of it belonging to the Kelvin waves. On day 14, only $10 \%$ of the response variance is due to the Kelvin waves. Although the $n=1$ Rossby mode is the main contributor to the Rossby variance at all time scales, the $n>1$ Rossby modes contribute over $50 \%$ of the balanced response to the MJO heating. In the short range, dipole perturbations produce a response with the maximal variance in zonal wavenumbers $k=2-3$ whereas in the medium range the response maximizes at $k=1$ in all experiments. Furthermore, the medium-range response to the heating perturbation mimicking MJO phase 6 is found also over Europe.
\end{abstract}

\section{Introduction}

The Madden-Julian oscillation (MJO) is the most important component of subseasonal variability in the tropics (Madden and Julian 1971, 1972). Its slow eastward movement during 30-90 days has been described by eight phases of heating perturbations over the Indian Ocean, Indonesia, and western equatorial Pacific (e.g., Madden and Julian 1972; Zhang 2005), some phases characterized by a heating monopole (e.g., phases 4 and 8) whereas in other phases heating perturbations appear more similar to heating dipoles

\footnotetext{
${ }^{\text {a }}$ Current affiliation: Meteorological Institute, University of Hamburg, Hamburg, Germany.
}

Corresponding author: Katarina Kosovelj, katarina.kosovelj@ fmf.uni-lj.si (e.g., phases 2 and 6) (e.g., Cassou 2008; Lin et al. 2009).

The global circulation response to MJO-like tropical heating perturbations is studied using numerical simulations employing models and heating perturbations with different levels of complexity. A common feature of the tropical response to localized heating perturbations consists of the upward motion over the heated region with divergence in the upper troposphere, driving the anomalous Walker circulation in the eastwest direction and convergence toward the heating in the lower troposphere. The resulting baroclinic vertical structure has been regularly identified in observations and reanalyses (e.g., Adames and Wallace 2014; Žagar and Franzke 2015). The zonal wind response along the equator together with two anticyclonic vortices north and south of the equator in the vicinity of the heating are known as "Gill type" response (Gill 1980) and are 
discussed in terms of the equatorial Kelvin wave and the equatorial Rossby wave with the lowest meridional mode (i.e., $n=1$ Rossby wave). The extratropical response to the tropical heating anomalies is composed of the barotropic Rossby wave train (Hoskins and Karoly 1981; Kasahara 1984; Kasahara and da Silva Dias 1986). Characteristics of the train depend on the location, season, sign, and longevity of the heating perturbations (Garcia and Salby 1987; Jin and Hoskins 1995; Park et al. 1995; Lee et al. 2009; Lin et al. 2010; Lin and Brunet 2018).

Although MJO is a time-varying phenomenon, studies of the response to a single (quasi stationary) MJO phase represented by a heating monopole or a dipole are valuable. Seo and Son (2012) reported that each pole of the dipole heating produces the Kelvin wave response to the east and the Rossby wave response to the west of the pole, in a similar manner as in the case of two heating monopoles. The responses from the two poles interact so that their geopotential height anomalies along the equator partially cancel. The extratropical response was studied by Lin et al. (2010) using both dipole and monopole temperature perturbations in a dry model. Their dipole perturbation produced the extratropical response in medium range, but the extratropical response to a monopole perturbation located over Indonesia was very weak. Park et al. (1995) and more recently Lin and Brunet (2018) compared responses to the dipolar perturbation with both combination of signs.

The present study extends previous research in several ways. We perform a series of numerical simulations using large ensembles and heating perturbations that realistically account for processes in state-of-the-art numerical weather prediction (NWP) models including feedbacks from the model convection, large-scale condensation schemes, and sea surface temperature (SST) on temperature tendencies. Our experiments include monopole and dipole heating perturbations over the Indian Ocean and Indonesia mimicking various phases of the MJO life cycle. The ensemble approach ensures that our simulations cover a broad range of background conditions. We carry out a scale-dependent quantification of the response to heating anomalies in terms of the Rossby waves and the inertio-gravity (IG) waves on the sphere by using the normal-mode function (NMF) decomposition (Kasahara and Puri 1981). The applied three-dimensional NMFs are orthogonal thus allowing us to quantify variance associated with each zonal horizontal and vertical scale of the Rossby and IG components of the response. The results provide a time development of the Rossby and IG wave variance spectrum in response to the stationary MJO-like heating perturbations. Modal analysis points out scales with strongest response in short and medium range.
The Kelvin wave and $n=1$ Rossby wave response to tropical heating perturbations has been the subject of several studies. Kasahara (1984) and Kasahara and da Silva Dias (1986) used NMFs to study the response to tropical heating perturbations in different background flows, and Zhong and Nogues-Paegle (1990) used NMFs to represent the response to the heating perturbation in the El Niño region, but none of these studies quantified the role of various IG and Rossby waves. Recently the NMF method was used by Žagar and Franzke (2015) by applying a linear regression between an MJO index and reanalysis data and they reported a dominant role of the spherical $n=1$ Rossby wave in the global variance associated with the MJO. Stechmann and Majda (2015) analyzed the MJO in terms of the Kelvin and Rossby $n=1$ on the equatorial $\beta$ plane. In contrast to the $\beta$ plane, where the equatorial wave trapping is ensured by the equatorial Rossby radius of deformation (i.e., by the applied equivalent depth), the equatorial confinement on the sphere is additionally controlled by the zonal wavenumber and even the barotropic Kelvin wave with equivalent depth around $10 \mathrm{~km}$ on the sphere is confined within the tropical belt (Boyd and Zhou 2008). The present study thus quantifies the Rossby and inertiogravity wave response to the MJO-like heating perturbations including nonlinear, moist feedbacks, as a function of the horizontal scale and response time.

The outline of the paper is as follows. Section 2 presents the new ensemble perturbation method, setup of numerical experiments, and NMF decomposition. Results regarding the response to the monopole and dipole heating perturbations are presented in section 3 . The summary and conclusions are given in section 4 .

\section{Methodology}

\section{a. Numerical model}

We perform ensembles of numerical simulations using the International Centre for Theoretical Physics (ICTP) AGCM, also known as the Simplified Parameterization, Primitive Equation Dynamics (SPEEDY) model (Molteni 2003; Kucharski et al. 2006, 2013). SPEEDY is a hydrostatic spectral general circulation model (GCM) with vorticity and divergence as the prognostic variables, together with temperature, surface pressure, and specific humidity. Convection, condensation, clouds, radiation, momentum and energy surface fluxes, and vertical diffusion are assessed in simplified parameterization schemes, described in detail in Molteni (2003).

The 100-member ensemble described below is based on SPEEDY, version 41, with 8 terrain-following sigma levels, defined as $\sigma=\left(p-p_{t}\right) /\left(p_{s}-p_{t}\right)$, where $p_{s}$ is surface 
pressure and $p_{t}=0 \mathrm{hPa}$ the model-top pressure. The model levels are $\sigma=0.950,0.835,0.685,0.510,0.340$, $0.200,0.095$, and 0.025 . The horizontal discretization applies T30 spectral truncation including 30 waves along each latitude circle. The time step for integration was $20 \mathrm{~min}$. Outputs were saved as daily averages in gridpoint space on an N24 Gaussian grid.

\section{b. Formulation of the ensemble}

To prepare a large ensemble, an unperturbed continuous simulation was first integrated for 102 years, from 1909 to 2010. It was forced by SST from the ECMWF twentieth-century reanalysis (ERA-20C) (Poli et al. 2016). The monthly mean SST was prescribed on day 15 of a month, then linearly interpolated to daily values. This is the control simulation. Starting with the third year (year 1911) of the control run, the model was restarted every 1 January with a continuous additional heating perturbation and it was run for 3 months. These 100 integrations represent the ensemble members.

To simulate realistic MJO-like heating perturbations, we included all the factors known to influence the MJO heating perturbations: convection, largescale condensation, and SST. Heating perturbations are added to temperature tendencies in each grid point, and are calculated as

$$
\left(\frac{\partial T}{\partial t}\right)_{\text {pert }}(\lambda, \phi, \sigma)=F_{\mathrm{SST}} H_{\text {pert }}(\lambda, \phi)\left(\frac{\partial T}{\partial t}\right)_{\mathrm{CC}}(\phi, \sigma) .
$$

The first factor in (1) is the SST-dependent function $F_{\text {SST }}$ that defines whether SST can trigger convection. If SST $>\mathrm{SST}_{\text {crit }}$ then

$$
F_{\text {SST }}=k_{s}\left(\mathrm{SST}-\mathrm{SST}_{\text {crit }}\right)
$$

and $F_{\mathrm{SST}}=0$ otherwise. The critical SST, $\mathrm{SST}_{\text {crit }}=24^{\circ} \mathrm{C}$. Given the average distribution of SST, this parameterization ensures the meridional confinement of the heating perturbations between $30^{\circ} \mathrm{S}$ and $30^{\circ} \mathrm{N}$. The tuning constant $k_{s}=0.2$ was chosen empirically to couple the SST difference with the model convection scheme.

The second term $H_{\text {pert }}$ defines the zonal pattern of the heating perturbation as $H_{\text {pert }}= \pm k_{h} \cos (3 \lambda) \operatorname{rand}(0,1)$, where $\pi / 6 \leq \lambda \leq 5 / 6 \pi$ for dipole and $\pi / 2 \leq \lambda \leq 5 / 6 \pi$ for the monopole heating. Depending whether the sign of $H_{\text {pert }}$ is positive or negative, the perturbation represents the heating or the cooling, respectively. The tuning constant $k_{h}=10$ was used to increase the amplitude of heating close to the one in previous studies and in order to get statistically significant response throughout the whole troposphere. The horizontal patterns of $H_{\text {pert }}$ are multiplied at every time step with 0 or 1 at each grid point in a random a) $\mathrm{M} /-$

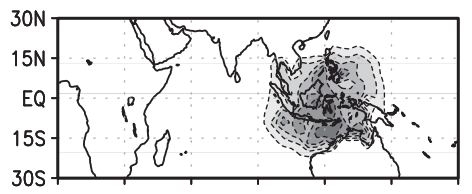

b) $\mathrm{D}+/-$

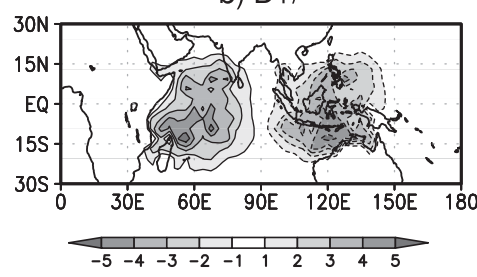

c) vertical profile

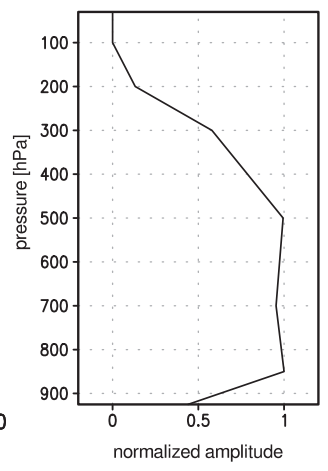

FIG. 1. Averaged heating pattern at $500 \mathrm{hPa}$, for (a) monopole $(M /-)$ and (b) dipole $(D+/-)$ experiments. The $D-/+$ and $M /+$ have similar patterns but opposite signs. Contours are every $1 \mathrm{~K} \mathrm{day}^{-1}$ with negative values dashed. (c) Time-averaged vertical profile of heating perturbation, normalized by the amplitude at $500 \mathrm{hPa}$.

manner [represented by rand $(0,1)$ ] to simulate the spatial variability of the MJO pattern. This is a crude representation of convection. The last term in $(1),(\partial T / \partial t)_{\mathrm{CC}}$, represents zonally averaged temperature tendencies at model levels due to convection and large-scale condensation.

The list of experiments includes two dipole experiments and several experiments with monopole heating or cooling perturbations. The dipole heating perturbation that has a maximum over the Indian Ocean and a minimum over Indonesia (Fig. 1b) will be referred to as $D+/-$. The opposite dipole (with minimum over Indian Ocean and maximum over Indonesia) is denoted $D-1+$. The two poles are centered at $60^{\circ}$ and $120^{\circ} \mathrm{E}$. The monopole perturbation that is made only of the cooling over Indonesia will be referred to as $M /-$ (Fig. 1a). The heating monopole over Indonesia will be called $\mathrm{M} /+$.

Figures $1 \mathrm{a}$ and $1 \mathrm{~b}$ shows the structure of timeaveraged perturbations at $500 \mathrm{hPa}$ for $D+/-$ and $M /-$ experiments. The $D-/+$ and $M /+$ experiments have similar shape and opposite signs than $D+/-$ and $M /-$. The average vertical profile of heating perturbations is shown in Fig. 1c, normalized by the heating amplitude at $500 \mathrm{hPa}$. It shows that the heating at $300 \mathrm{hPa}$ is about half of that at $500 \mathrm{hPa}$ and drops to a small value at $200 \mathrm{hPa}$ and to zero above the tropopause. The average amplitude of the heating/cooling is up to about $5 \mathrm{~K} \mathrm{day}^{-1}$, but the instantaneous gridpoint amplitude can be greater.

The described heating perturbation is further modified by the positive feedback through convection and condensation as the prescribed heating (cooling) perturbations locally increase (decrease) moist air convection. As a consequence, the condensation rates increase (decrease), which results in an increased (decreased) latent heat 
release and additional heating (cooling). The effect was not represented in the previous studies addressing the circulation response to tropical heating perturbations.

\section{c. Evaluation of the response}

The circulation response is analyzed in physical and spectral space.

\section{PHYSICAL SPACE}

Results in physical space are evaluated in terms of the ensemble-averaged response defined as

$$
\bar{A}(t)=\frac{1}{J} \sum_{j=1}^{J}\left[A_{j}(t)-A_{0}(t)\right],
$$

where $A_{j}$ is the analyzed variable of the $j$ th ensemble member and $A_{0}$ is the control value. Three studied variables are the geopotential height, zonal wind, and meridional wind. The parameter $t$ stands for the day after the start of integration.

The responses computed with (2) were tested for statistical significance with the $t$ test for unequal variances, the Welch $t$ test (Welch 1947). The null hypothesis, that the mean geopotential of the perturbed simulations is equal to the mean geopotential of control simulation, was tested for $95 \%$ confidence interval in each grid point for all discussed fields. The results are statistically significant.

The presentation focuses on day 3 of the integrations, representing the short-range response, and on day 14 , representing the medium-range response. The upper and lower troposphere are represented by levels 200 and $850 \mathrm{hPa}$, respectively.

\section{d. Modal decomposition}

The NMF decomposition represents the model outputs in terms of the eigensolutions of the linearized primitive equations (Kasahara and Puri 1981; Phillips 1990): the Rossby waves and the inertio-gravity waves. The decomposition is performed using the MODES package (Žagar et al. 2015b). Winds and transformed geopotential height data on $\sigma$ levels are expanded in terms of the Rossby and IG modes as

$(u, v, h)^{\mathrm{T}}=\sum_{m=1}^{M} \mathbf{S}_{m}\left[\sum_{n=1}^{R} \sum_{k=-K}^{K} \chi_{n}^{k}(m) \mathbf{H}_{n}^{k}(m)\right] G_{m}(\sigma)$.

The three elements of the input data vector in (3) are defined at a horizontal point $(\lambda, \varphi)$ of the $j$ th $\sigma$ level. The three indices of the complex multivariate expansion coefficients $\chi_{n}^{k}(m)$ are the zonal wavenumber $k$, the meridional index $n$, and the vertical mode index $m$. The basis structure functions in the vertical direction are denoted $G_{m}$, with $m$ varying from 1 to the maximal

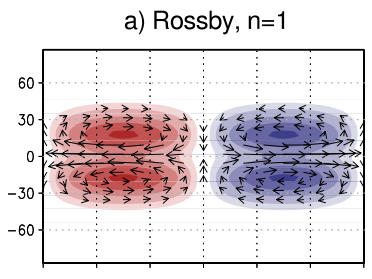

c) Kelvin wave

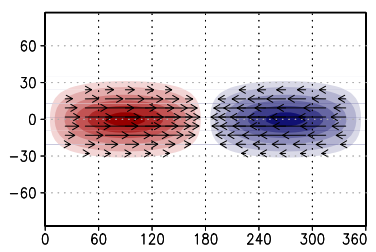

FIG. 2. Horizontal structure of Hough mode nondimensional wind and geopotential height for several meridional modes: (a) $n=1$ Rossby wave, (b) $n=2$ Rossby wave, (c) Kelvin wave ( $n=0$ EIG mode), and (d) $n=3$ Rossby wave. All examples are for the vertical mode $m=4$ and zonal wavenumber $k=1$.

number of model levels $M=8$. The first vertical structure function has no change of sign (barotropic) whereas the remaining seven have between one and seven zero crossings in the vertical (baroclinic structure). The Hough harmonics $\mathbf{H}_{n}^{k}(m)$ consist of waves in the zonal direction $[\exp (i k \lambda)]$ and the Hough vector functions in the meridional direction, which are composed of the associated Legendre polynomials (Kasahara 1976). The diagonal $3 \times 3$ matrix $\mathbf{S}_{m}$ assures that the input vector to the horizontal projection is dimensionless. Selected horizontal truncations correspond to the model discretization: $K=30$ and $R=90$. The meridional mode index defines two different types of solutions: westwardpropagating, low-frequency, Rossby-Haurwitz-type balanced modes (Rossby modes), and high-frequency IG modes, which propagates eastward (EIG) and westward (WIG). For our experiments 30 Rossby, 30 EIG, and 30 WIG modes were applied in (3) and therefore $R=90$. The meridional modes in each group (i.e., Rossby, EIG, and WIG modes) take indices 0-29. For details of the modal decomposition procedure the reader is referred to Žagar et al. (2015b) and references therein.

Selected modes or their combination can be filtered to physical space to describe their spatial characteristics with respect to the full fields. This is achieved by the inverse of (3). Two modes of particular interest in this article are the $n=1$ Rossby mode and the EIG mode with the lowest meridional index, that is, the Kelvin wave $(\mathrm{KW})$. The horizontal structures for several modes are shown in Fig. 2 for $k=1$ and $m=4$. The associated equivalent depth is $328 \mathrm{~m}$. As illustrated in Fig. 2, Rossby modes with odd values of $n$ are symmetric, whereas even $n$ Rossby modes are antisymmetric in their 
zonal wind and geopotential height fields with respect to the equator. The opposite applies to the IG modes. The $\mathrm{KW}$ ( $n=0$ EIG mode) is a symmetric mode.

In NMF space, the response is studied in terms of modal variances that are computed as

$$
V_{n}^{k}(t)=\frac{1}{J} \sum_{j=1}^{J} \sum_{m=1}^{M} g D_{m}\left|\chi_{n}^{k}(m ; j, t)-\chi_{n}^{k}(m ; 0, t)\right|^{2},
$$

where $j$ counts ensemble members $(j=0$ represents the control run), and $g D_{m}$ is the scaling factor which provides the variance $V$ in units of energy per unit mass $\left(\mathrm{J} \mathrm{kg}^{-1}\right)$. The equivalent depth for $m$ th vertical mode is denoted $D_{m}$ while $g$ stands for gravity. The 3D orthogonality of modal decomposition provides the total variance as the sum of variances in all modes. Following Žagar et al. (2015a), it can be easily shown that the integrated $V$ is equivalent to the sum of variances of the winds and transformed geopotential height in physical space after the vertical projection.

\section{Rossby and inertio-gravity mode response to tropical heating anomalies}

The results section will focus on the variance spectra [(4)] of the global response to monopole and dipole heating perturbations. But first we show the total response filtered in the Rossby and IG contributions to provide the picture of basic structures in physical space for the discussion.

\section{a. Near- and far-field response in Rossby and inertio-gravity modes}

\section{1) SHORT-TERM RESPONSE}

The short-term total response to a heating perturbation (Figs. 3a,g) over Indonesia resembles the well-known Gill-type response (Gill 1980) with easterlies over the western Pacific in the lower troposphere (Fig. 3g) and westerlies at $200 \mathrm{hPa}$ (Fig. 3a). When the 3-day response in the $M /+$ experiment is split in the Rossby and IG parts, the latter makes the most of the response within $\pm 20^{\circ}$ off the equator and it has the structure of the Kelvin wave (Figs. 3c,i). The statistically significant part of the 3-day response includes also the balanced vortices north and south of the equator with the maximal amplitude over Southeast Asia at around $30^{\circ} \mathrm{N}$ slightly west of the heating maximum at $120^{\circ} \mathrm{E}$. Clearly, the short-range tropical IG response has a stronger amplitude than the response projecting on the Rossby modes. Notice also in Fig. 3 that the statistically significant response in the lower troposphere over the Indian Ocean, Africa, and eastern Pacific is unbalanced (IG type) whereas in the upper troposphere both balanced and unbalanced components in these areas are statistically significant.

The response to a dipole perturbation $\mathrm{D}-/+$ is approximately a sum of responses to two distinct monopole perturbations with opposite signs, located $60^{\circ}$ longitude apart (Figs. 3d,j). Each pole produces the Kelvin wave-dominant response to the east and the Rossby wave response to the west of the pole and the two responses along the equator partially cancel (e.g., Seo and Son 2012). The experiments $M /-$ and $D+/-$ produce response patterns with opposite signs compared to $M /+$ and $D-/+$, respectively (not shown). The amplitude of the tropical response is comparable between the four experiments. An important difference between the monopole and dipole cases is in a greater amplitude of the Rossby wave response in the subtropics (Fig. 3e) and its localization in the case of dipole perturbations. Statistically significant impact outside the perturbed region in the upper troposphere in $D+/-$ is limited to the initialization of the Rossby wave over Southeast Asia in Fig. 3e. This suggests that the MJO phases with monopole shape of heating or cooling perturbations impact wider tropical regions in short-range forecasts in comparison to perturbations with dipole shape, due to equatorially trapped large-scale circulation response to the heating perturbations. This property will be quantified in modal space with the response variance spectra.

\section{2) MEDIUM-RANGE RESPONSE}

With time the response grows and travels out of the heated region. Statistically significant extratropical Rossby wave train reaches North America in 8-10 days, a result in agreement with previous studies. On day 14 , the $D-/+$ and $M /+$ experiments produce the most similar response among the four experiments (Fig. 4). The differences between the two are in the amplitude with $D-/+$ having a stronger response and in the response pattern. The presence of the cooling pole in $D-/+$ somewhat enhances upper-troposphere westerly wind response over Africa and the western Indian Ocean and easterly wind response over the Indian subcontinent with respect to $M /+$ (Fig. $4 \mathrm{~g}$ ). This is also seen in a stronger and more northward-directed response over the Middle East and Europe (Fig. 4g vs Fig. 4e). This westward component of response reaches Europe by day 10 (figure not shown). Another extratropical difference between $M /+$ and $D-/+$ is a stronger impact over the northern Pacific, especially in the Gulf of Alaska in $D-/+$ (Fig. 4g).

The $D+/-$ total response differs significantly from $D-/+$. First of all, there is no statistically significant response in the upper troposphere over the tropical 
a) $200 \mathrm{hPa}, \mathrm{M} /+$, total response

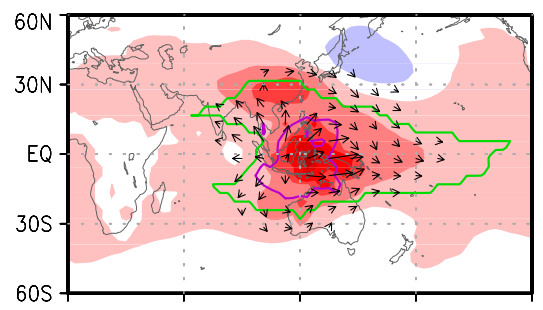

d) $200 \mathrm{hPa}, \mathrm{D}-/+$, total response

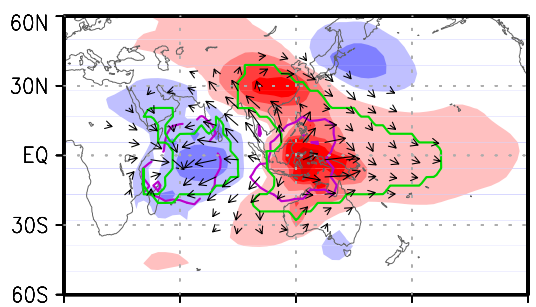

g) $850 \mathrm{hPa}, \mathrm{M} /+$, total response

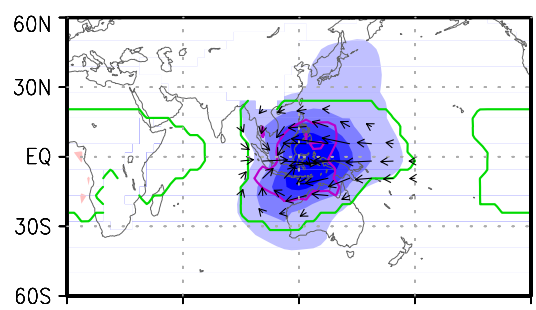

j) $850 \mathrm{hPa}, \mathrm{D}-/+$, total response

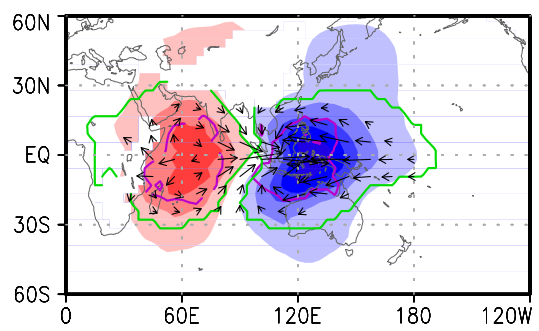

b) $200 \mathrm{hPa}, \mathrm{M} /+$, Rossby

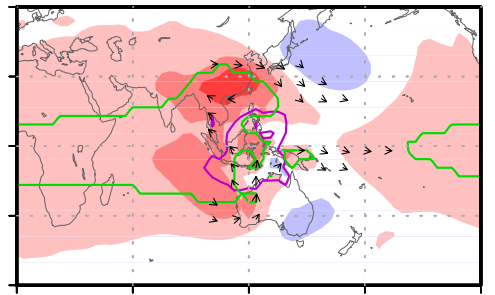

e) $200 \mathrm{hPa}, \mathrm{D}-/+$, Rossby

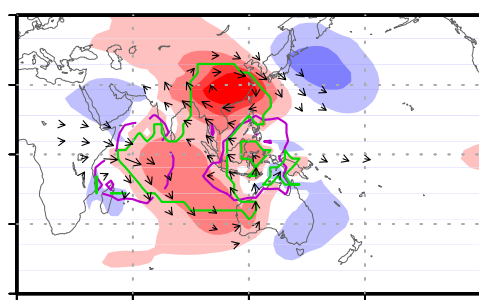

h) $850 \mathrm{hPa}, \mathrm{M} /+$, Rossby

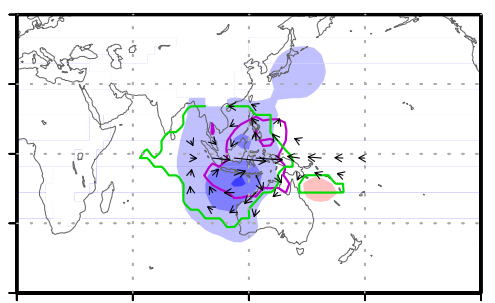

k) $850 \mathrm{hPa}, \mathrm{D}-/+$, Rossby

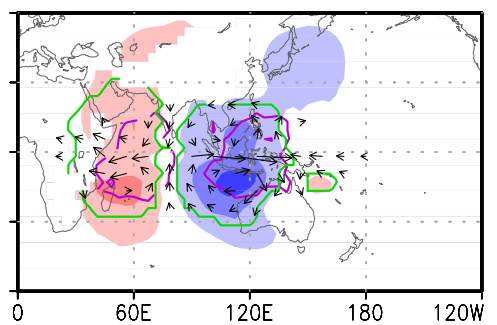

c) $200 \mathrm{hPa}, \mathrm{M} /+, \mathrm{IG}$

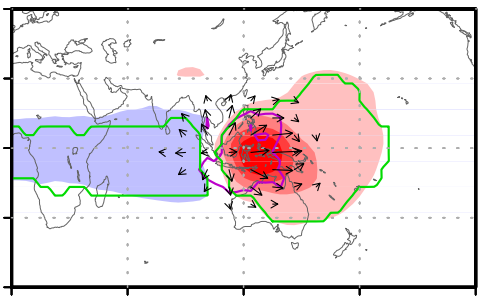

f) $200 \mathrm{hPa}, \mathrm{D}-\mathrm{I}+$, IG

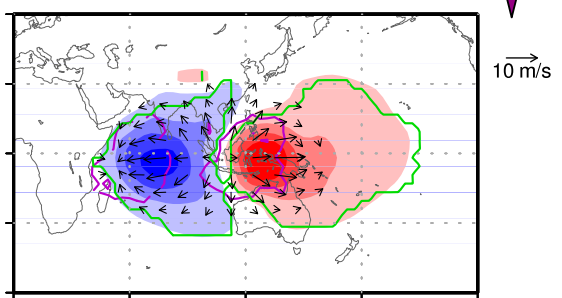

i) $850 \mathrm{hPa}, \mathrm{M} /+$, IG

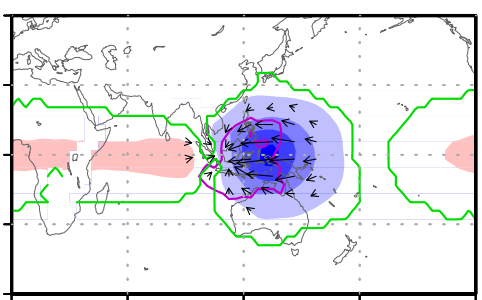

I) $850 \mathrm{hPa}, \mathrm{D}-/+$, IG

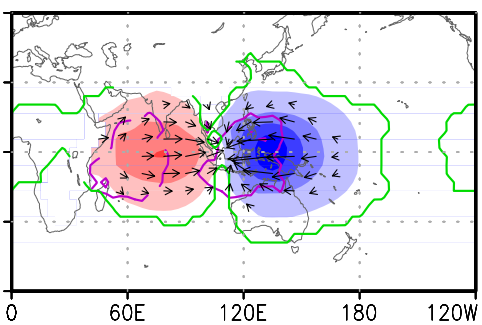

FIG. 3. Response to the two heating perturbations on day 3 separated in Rossby and IG components: (a)-(c),(g)-(i) $M /+$ and (d)-(f),(j)-(l) $\mathrm{D}-/+$ at (a)-(f) 200 and (g)-(l) $850 \mathrm{hPa}$. (a),(d),(g),(j) Total response, (b),(e),(h),(k) response in Rossby modes, and (c),(f),(i),(l) response in IG modes. The geopotential height is shown in meters (color shading) and wind (vectors) is in meters per second. The patterns of $M /-$ and $D+/-$ are similar, but with opposite sign. Purple lines represent the contour of the $\pm 2 \mathrm{~K}^{2} \mathrm{day}^{-1}$ heating perturbation from Fig. 1. Green lines are borders of the statistically significant response. Wind vectors are drawn only for speeds stronger than $1.5 \mathrm{~m} \mathrm{~s}^{-1}$.

Pacific east of the negative pole over Indonesia (Fig. 4c). Another difference appears in the Rossby wave train that is more zonal and has a larger amplitude in $D+/-$ than in $D-/+$. The maximal wave response over East Asia and Japan in $D+/-$ is a superposition of the Rossby wave trains emanating from the subtropical Rossby waves initiated by the two heating anomalies, as reported by Seo and Lee (2017). This is confirmed by an additional experiment with a positive monopole over the Indian Ocean (not shown). In $D+/-$, the response to the two monopoles cancels over most of the tropics.
Among the four experiments, the response is weakest in $M /-$ that has similar amplitudes of the response in the tropical and extratropical fields (Fig. 4a). A stronger response to the heating perturbation than to the cooling is the reason for the observed similarities in the response to $M /+$ and $D-/+$ perturbations. The same applies to the $D+/-$ and a heating monopole over the Indian Ocean (not shown). Hence the location of the positive heating anomaly plays an important role in the response pattern. A similar result was showed by Lin et al. (2010) and Ting and Sardeshmukh (1993) for their monopole heating. 
a) M/-, total response

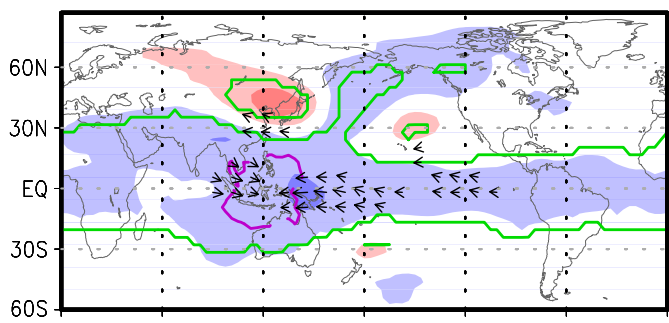

c) D+/-, total response

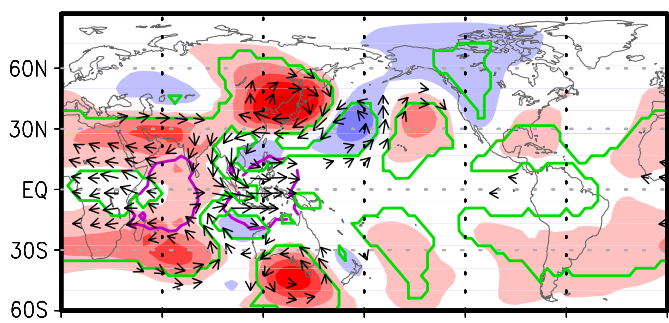

e) $\mathrm{M} /+$, total response

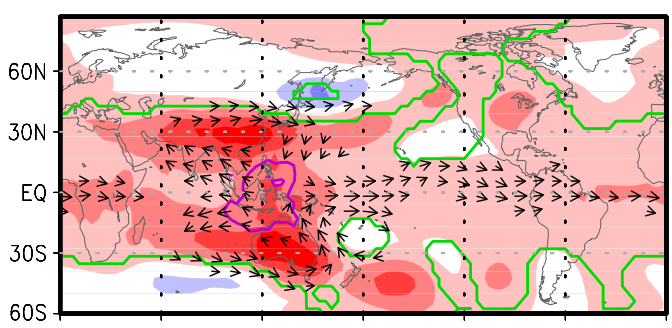

g) D-/+, total response

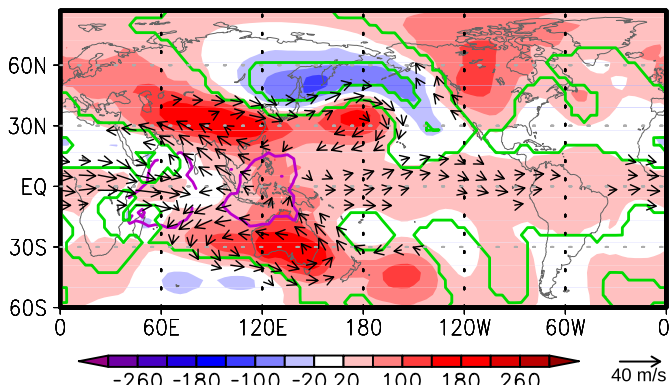

b) $M /-$, IG

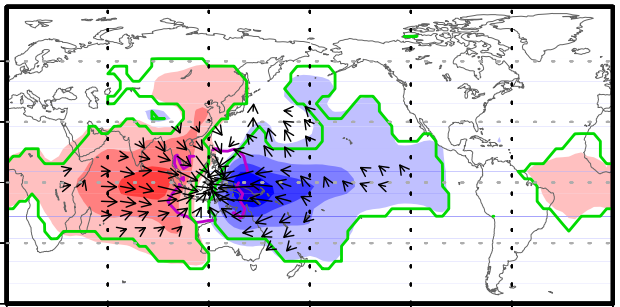

d) $D+/-$, IG

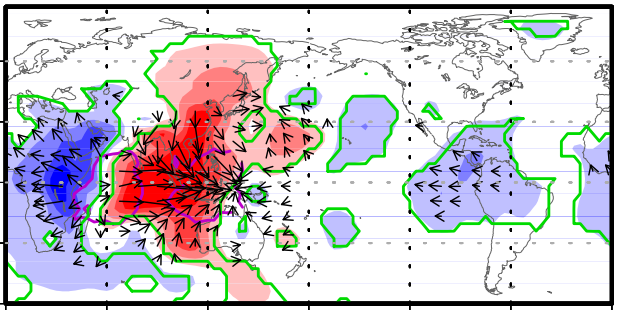

f) $M /+, I G$

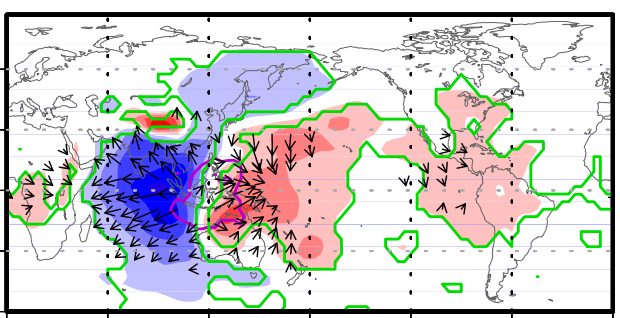

h) $D-/+, I G$

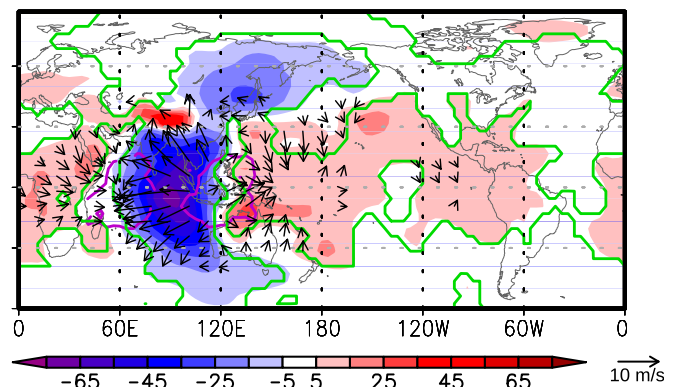

FIG. 4. As in Fig. 3, but for the (a),(c),(e),(g) total and (b),(d),(f),(h) IG response on day 14 for (a),(b) $M /-$, (c),(d) $D+/-$, (e),(f) $M /+$ and (g),(h) $D-/+$ experiments at $200 \mathrm{hPa}$. Wind vectors are drawn only for speeds stronger than $7 \mathrm{~m} \mathrm{~s}^{-1}$ for the total response and $1.5 \mathrm{~m} \mathrm{~s}^{-1}$ for the IG response.

Figure 4 includes also the IG component of the 14-day response. Notice that the medium-range total winds are scaled by factor 4 in comparison with the IG winds (Fig. 3 and 4b,d,f,h). The tropical IG response in medium range is more complex compared to day 3 due to interaction between convection in the vicinity of the heating perturbations with propagating IG waves. Convection produces a strong inflow (outflow) over the cooling (heating) region in the upper troposphere. The superposition of the IG response to the two poles results in no impact on the central Pacific circulation in the upper troposphere in $D+/-$ (Figs. 4c,d). The interaction of the outflow from the heating pole centered at $120^{\circ} \mathrm{E}$ with the Himalayan relief in our $\sigma$-level model produces a stationary wave response in the IG component seen in Figs. 4f and 4h. In the extratropics, the maximal IG response is found in the vicinity of the strongest upper-tropospheric jet (not shown). This ageostrophic circulation response is due to the interaction of the traveling response with the westerly jet. 
The amplitude of the IG part response over eastern Asia reaches up to $25 \%$ of the total amplitude of the response in this region. Finally, Fig. 4 also shows that monopole perturbations over Indonesia produce no IG response over northern Africa, the Middle East, and Europe.

\section{b. Modal view of the global response to tropical heating anomalies}

\section{1) VARIANCE GROWTH}

The time development of the global-response variances in the four experiments is shown in Fig. 5a. The four simulations are complemented by an additional experiment denoted $M+/$ with a heating over the Indian Ocean (thin gray line in Fig. 5a). Initially the dipoles induce more variance in response to larger absolute perturbations than monopoles. As the simulation time increases, variance in the two experiments with the heating pole over Indonesia increases faster, and after day 5 the $D-1+$ experiment has the largest variance as could be expected based on Fig. 4. Similarly, the $M /$ - experiment has the smallest variance, in accordance to the weakest response in gridpoint space.

In the medium range, 1-2-weeklong response, there is a clear difference between the weak response experiment $M /-$ (thin black line in Fig. 5a), the strongest response in $D-/+$, and intermediate response in experiments $M /+$, $M+/$, and $D+/-$. A faster growth of variance in experiments with heating over Indonesia is probably a combination of several factors. One factor is a stronger convection-condensation feedback over the Indonesian heating perturbation in comparison with the Indian Ocean that is confirmed by comparing the precipitation rates and the $(\partial T / \partial t)_{\mathrm{CC}}$ term in (1) among the experiments. Anomalous convection and condensation heating are stronger as well as anomalous precipitation when heating is over Indonesia (not shown). Another factor may be the interaction with the background flow. A heating anomaly centered at $120^{\circ} \mathrm{E}$ is closer to the maxima of the westerly jet and the meridional shear of the zonal wind (maximal relative vorticity) than the heating anomaly centered at $60^{\circ} \mathrm{E}$, which supports a stronger interaction with the jet (Sardeshmukh and Hoskins 1988; Webster and Chang 1998; Seo and Lee 2017). However, the longrange response is dominated by a continuously increased variance due to heating over the Indian Ocean.

Figure $5 \mathrm{~b}$ quantifies the portion of the IG variance in the total response to tropical heating anomalies. It shows that the initial response, concentrated in the tropics, is dominated by the IG variance at about $70 \%$ level. The variance equipartition is reached by day 5 in all experiments except $M /-$ in which it happens at day 3 . a) time development of total variance

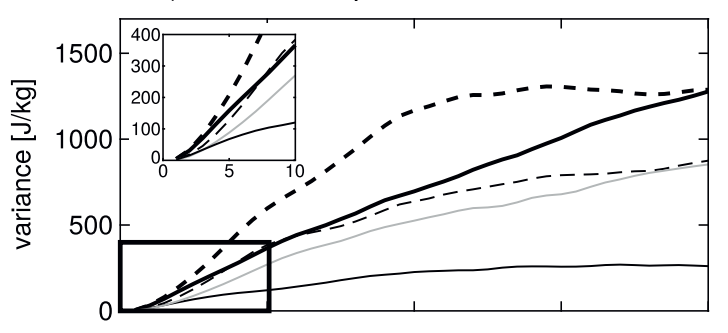

b) portion of Rossby and IG variance in total variance

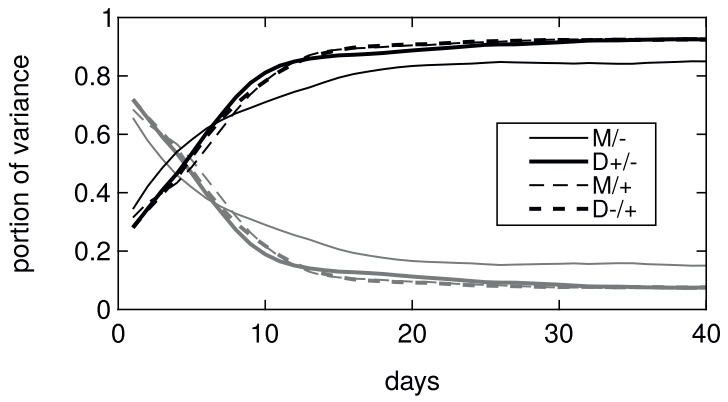

FIG. 5. Time development of (a) total variance (black lines) and (b) portion of the Rossby variance (black lines) and IG variance (gray lines). The thin gray line in (a) represents experiment with a positive heating perturbation over Indian Ocean.

As the response propagates into the midlatitudes, the Rossby modes variance increases and at day 14 , the percentage of the Rossby variance is $88 \%$ ( $78 \%$ in $M /-)$. In equilibrium, there is $93 \%$ of the global variance response in the Rossby modes except in the $\mathrm{M} /-$ case, which is characterized by $85 \%$ balanced variance in the long time range. In conclusion, although different anomalies induce responses with significantly different amplitudes and distinct structures, in each case the variance partition between the balanced and IG modes reaches the equilibrium state after about 3 weeks.

\section{2) ONE-DIMENSIONAL VARIANCE SPECTRA}

Figure 6 shows the distribution of the total global variance as a function of the zonal wavenumber. The difference between the dipole and monopole experiments on day 3, seen in Fig. 3 as a more local response in the $D-/+$ case, is illuminated in Fig. 6a. The two monopoles produce the maximal variance at $k=1$ at the level about $25 \%$ of the total variance and the variance decreases as the zonal wavenumber becomes larger. The response to dipoles is maximal at $k=3$ which contains approximately $30 \%$ of the total variance. The dipoles also have nearly the same percentage of variance in $k=2$, and a minimum at $k=6$. As discussed above, differences between the responses to dipole and monopole perturbations are due to interaction of the responses to the two 
a) day 3

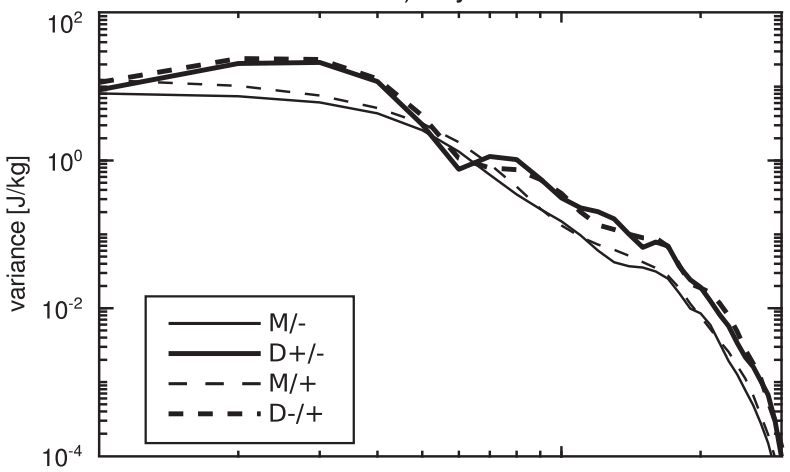

b) day 14

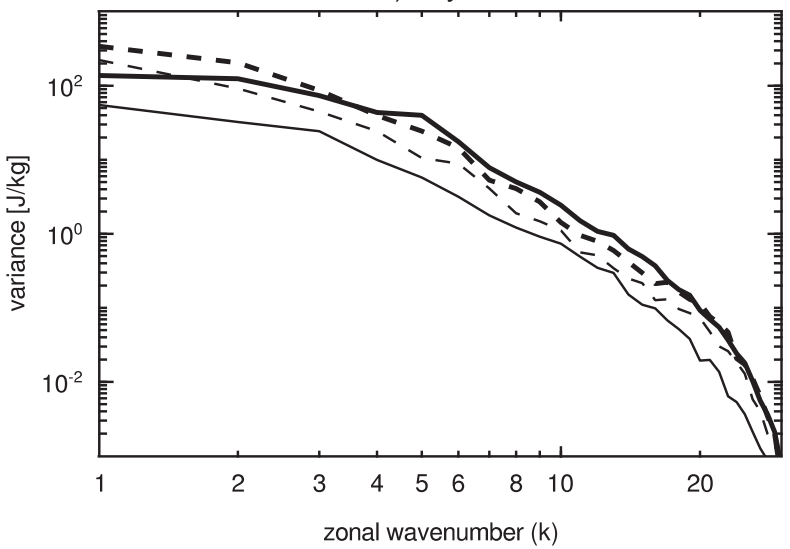

FIG. 6. Global variance distribution as a function of the zonal wavenumber $k$ on days (a) 3 and (b) 14 . The variance is meridionally and vertically integrated. The zonal mean state $(k=0)$ is not shown.

poles of the dipole. This interaction partially cancels the response in some wavenumbers while it amplifies wavenumbers close to the scale of forcing. The maximum in $k=3$ on day 3 is thus related to the horizontal scale of the dipole.

On day 14 (Fig. 6b), all experiments have the largest variance in $k=1$ although with different percentages of their respective total variances. For example, $D-/+$ has $47 \%$ of variance in $k=1$ and $11 \%$ in $k=3$ whereas $D+/-$ is characterized by $27 \%$ of variance in $k=1$ and $15 \%$ in $k=3$. This is due to a more homogeneous response in the zonal direction in the $D-1+$ case (see Fig. 4). The differences among experiments are influenced by the position of heating perturbations. A greater variance in $D+/-$ at wavenumbers $k=5-20$ reflects a stronger extratropical Rossby wave train presented in Fig. 4.

\section{3) TWO-DIMENSIONAL VARIANCE SPECTRA}

The distribution of variance among meridional modes for each zonal wavenumbers is presented in Fig. 7 at day 3 and in Fig. 8 at day 14. It quantifies predominant scales of the IG and Rossby mode responses. Notice that the $n=0$ Rossby mode for $k>0$ is the mixed Rossbygravity mode, associated with cross-equatorial winds whereas the mode $(n, k)=(0,0)$ is a constant without relevance for the discussion. Figures 7 and 8 point out the two prevalent meridional modes, the $n=1$ Rossby mode and the $n=0 \mathrm{IG}$ mode that is mostly contributed by the Kelvin waves, thus confirming previous studies. The zonally integrated percentages of the IG and Rossby variances are shown in Fig. 9.

Figures 7i-1 reveal a different distribution of the Kelvin wave variance over wavenumbers in dipole and monopole experiments. The maximum in the monopole case is found in $k=1$ whereas the dipoles have the maximal variance in $k=2-3$. This is similar to the $n=1$ Rossby mode presented in Figs. 7e-h. The 2D variance distributions in $D+/-$ and $D-/+$ have peaks in $k=3$ and the same $k$ also has relatively more variance than neighboring $k$ in the asymmetric mode $n=2$. The largest portion of variance in $k=0$ has the $M /+$ experiment (Fig. $7 \mathrm{~g}$ ).

In the medium range, there is a greater variance portion in higher modes, primarily in the Rossby modes (Fig. 8). All four experiments have the Rossby variance maximum in $(k, n)=(1,1)$ although the extended maxima in some experiments include also $k=0$. The amount of variance is larger in symmetric modes. On day 14 , there is between $75 \%$ and $85 \%$ of the Rossby variance in odd Rossby modes and about $90 \%$ of the IG variance in even IG modes. This confirms earlier idealized simulations of Kasahara (1984) and Kasahara and da Silva Dias (1986) who reported that the equatorially symmetric tropical heating preferably excites symmetric modes, regardless of the background flow. The asymmetric component in our case can for example be noticed in an increased variance in $n=2$ Rossby modes in $D-1+$ (Fig. 8h). Notice also in Fig. 2 that a phase collocation of the $n=1$ and $n=2$ Rossby modes with a similar vertical structure acts to enhance the asymmetry in physical space.

The Kelvin wave variance accounts for approximately $85 \%$ of the IG variance on day 3 and $70 \%-80 \%$ on day 14 . On day 3, the Kelvin wave contribution to the total variance varies from $45 \%$ in $M /-$ to $55 \%$ in the other experiments. This drops to approximately $15 \%$ of total variance in the $M /-$ experiment and $10 \%$ in other experiments on day 14 . The percentage of the Kelvin variance in the IG part remains approximately between $70 \%$ and $80 \%$ in the long range (Fig. 9a). An increase in the Kelvin wave variance from the start until day 5 followed by a variance reduction until day 9 may be due to the adjustment process to the imposed heating with the Kelvin waves transporting energy out of the perturbed region. The maximal Kelvin wave variance during this period is in the vertical mode $m=4$ with the equivalent depth $D_{4}=328 \mathrm{~m}$. 
a) all modes, M/-

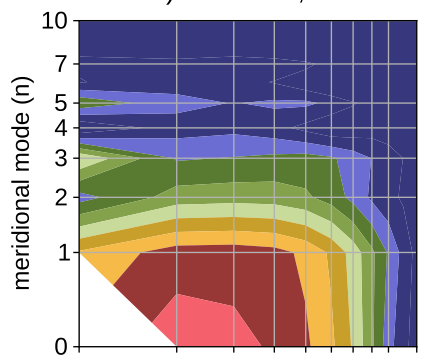

e) Rossby modes, M/-

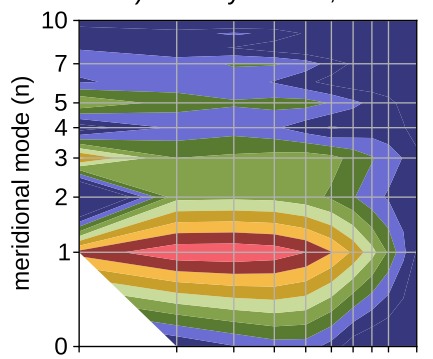

i) IG modes, M/-

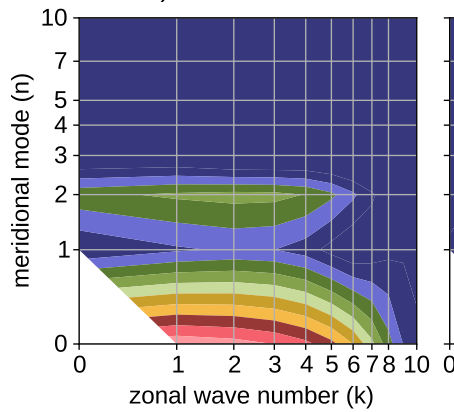

b) all modes, D+/-

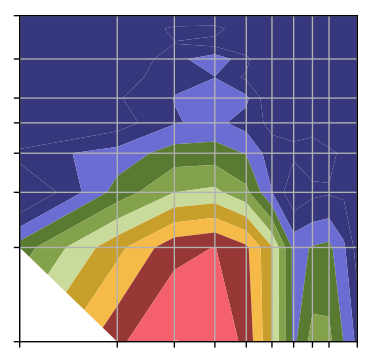

f) Rossby modes, D+/-

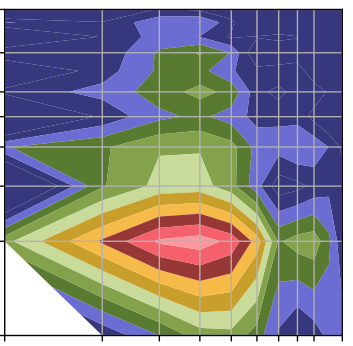

j) IG modes, D+/-

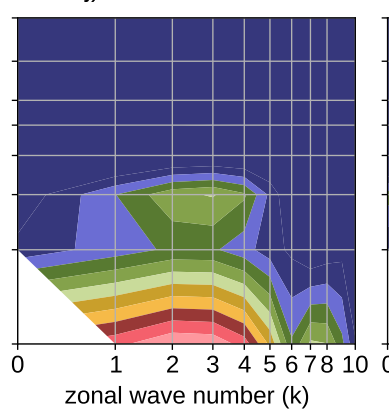

c) all modes, $\mathrm{M} /+$

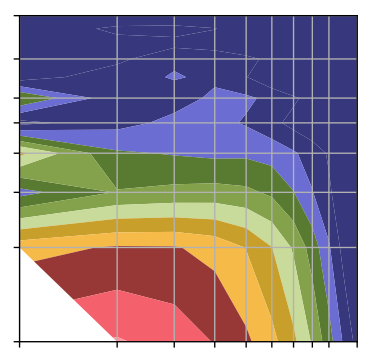

g) Rossby modes, M/+

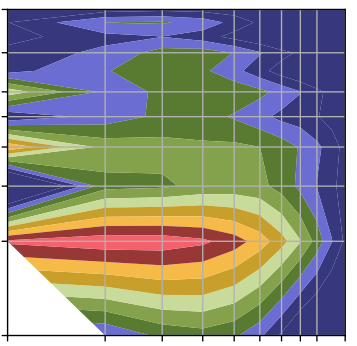

k) IG modes, M/+

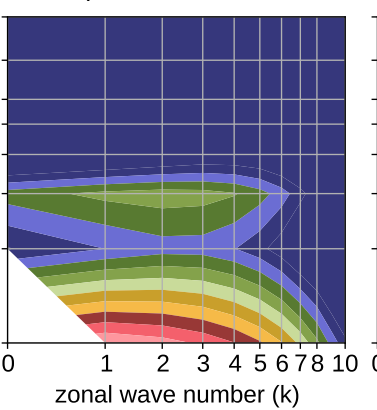

d) all modes, D-/+

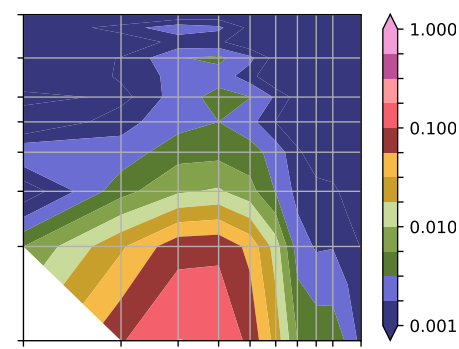

h) Rossby modes, D-/+

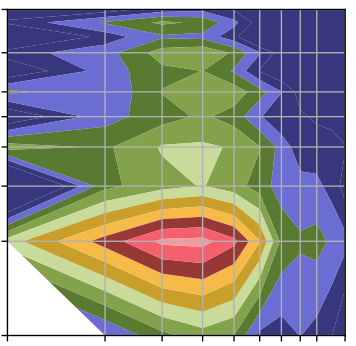

I) IG modes, D-/+

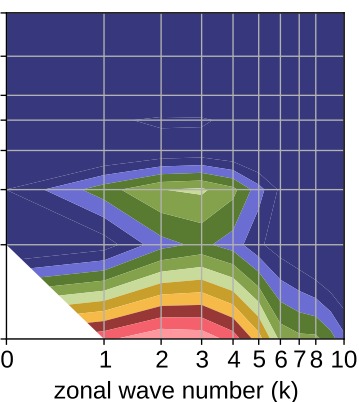

FIG. 7. Global variance distribution in response to heating perturbations on day 3 as a function of the zonal wavenumber and the meridional mode index $n$. The variance in each $(k, n)$ is divided by the integrated variance, $\sum_{k} \sum_{n} V_{n}^{k}$, over (a)-(d) all modes, (e)-(h) Rossby modes, and (i)-(l) IG modes. Each experiment applies normalization by the own total variance: (a),(e),(i) $M /-$, (b),(f),(j) $D+/-,(\mathrm{c}),(\mathrm{g}),(\mathrm{k}) \mathrm{M} /+$, and (d),(h),(l) $D-/+$. Only the lowest 10 zonal wavenumbers and meridional modes are shown.

On day 3 , the $n=1$ Rossby mode constitutes about $80 \%$ of the Rossby variance (Fig. $9 \mathrm{~b}$ ) that makes between $25 \%$ and $35 \%$ (in $M /-$ ) of the total variance. On day 14 , between $30 \%$ and $45 \%$ of the total variance and between $40 \%$ (dipoles) and 50\% (monopoles) of the Rossby variance is in the $n=1$ Rossby mode. In longer range, the portion of $n=1$ Rossby mode in the Rossby variance is $20 \%-45 \%$, with the experiment $M /-$ having the largest portion. This tells us that, on average, the higher $(n>1)$ Rossby modes contain the majority of the variance associated with the balanced circulation response to the tropical heating perturbations in the medium- and longer-range forecasts.

\section{Summary and conclusions}

We performed ensembles of simulations with MJOlike heating perturbations in 100 winters with different background conditions, including the vertical shear and the position of the Northern Hemisphere jets. The perturbations were applied on temperature tendencies and included feedbacks from the model convection and large-scale condensation schemes. This complex setup of numerical experiments questioned whether previous studies using dry models provided sufficient realism of the tropical and extratropical response to localized heating perturbations. Responses to dipole and monopole heating perturbations that mimic various phases of the MJO life cycle were compared to previous studies. The outputs were decomposed into Rossby and inertio-gravity (IG) wave components using the 3D orthogonal normal-mode function representation. The scale-dependent decomposition highlighted differences in spectral response to heating among various experiments and quantified transient properties of the Rossby and inertio-gravity mode response. 
a) all modes, M/-

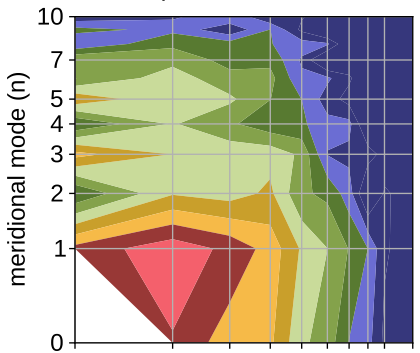

e) Rossby modes, M/-

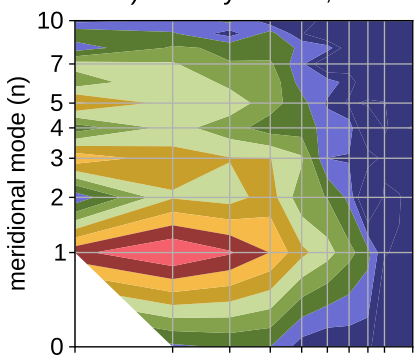

i) IG modes, M/-

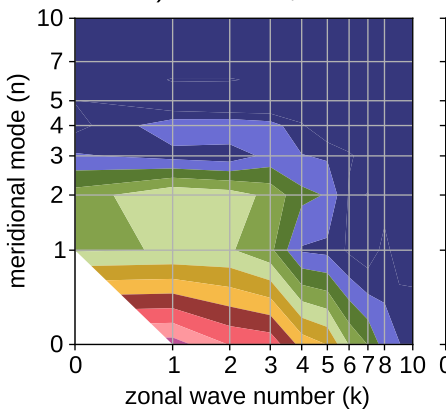

b) all modes, $D+/-$

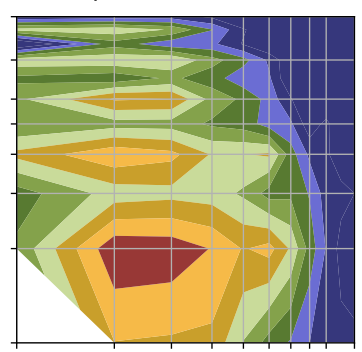

f) Rossby modes, D+/-

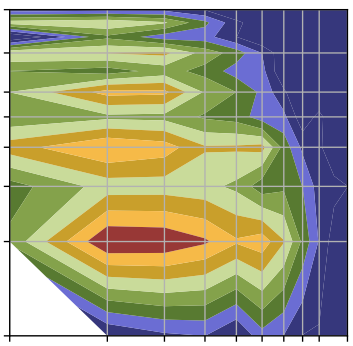

j) IG modes, D+/-

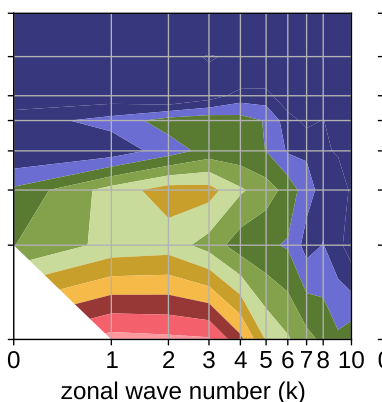

c) all modes, M/+

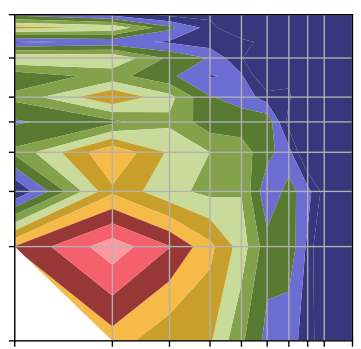

g) Rossby modes, M/+

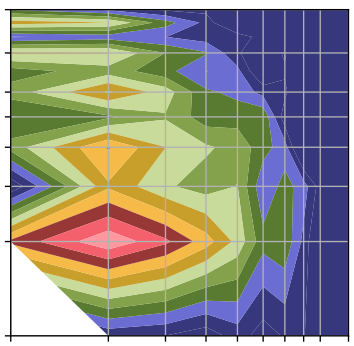

k) IG modes, M/+

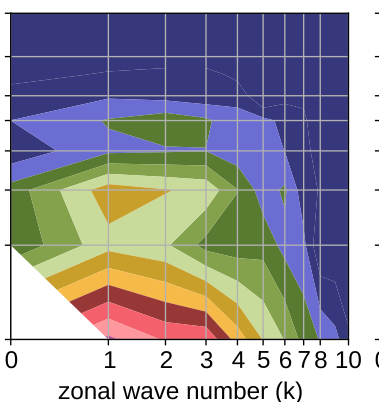

d) all modes, D-/+

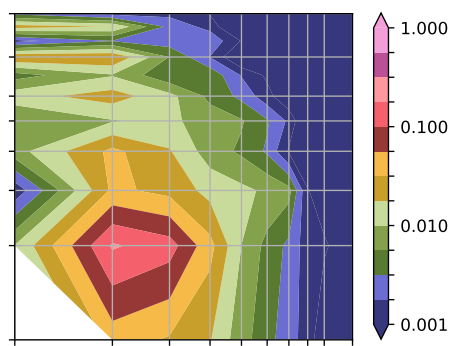

h) Rossby modes, D-/+

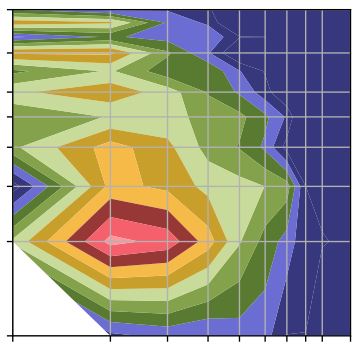

I) IG modes, D-/+

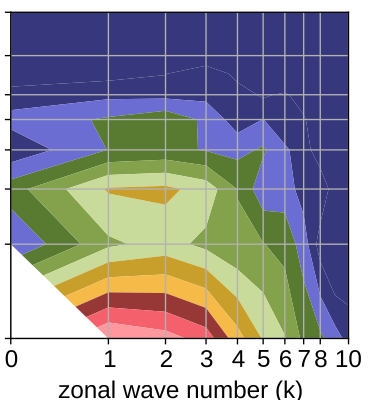

FIG. 8. As in Fig. 7, but on day 14.

The most relevant findings are as follows:

1) The realistic formulation of heating perturbations involving moist feedbacks does not produce significantly different results from a dry model with imposed temperature perturbations. Moist results strengthen the finding by Lin and Brunet (2018) regarding the westward-propagating response from heating in both Indian and Indonesian regions. In particular, a statistically significant response from the dipole heating perturbations mimicking the MJO phase 6 is found over Europe in the medium range. Our result suggesting that some phases of MJO can affect European weather on 10-day time scale through northwestward-propagating response requires further studies employing more realistic NWP models.

2) Although the response is mostly defined by a positive heating anomaly, the response to dipole perturbations is still approximately a sum of responses to heating and cooling poles located over the Indian Ocean and Indonesia. The interaction of the two poles acts to localize the tropical response and to affect the shape and orientation of the Rossby wave emanating from the subtropics. The effect on the short-range variance distribution of the response is seen in the maximal variance in zonal wavenumbers $k=2-3$ for dipole heating in contrast to monopole heating that produces the peak response in $k=1$. In medium range, all perturbations have the largest response in $k=1$ but dipoles produce relatively larger variance in synoptic scales reflecting a stronger Rossby wave train signal in the extratropics. The zonal mean state $(k=0)$ response has a significant percentage of variance $(10 \%-15 \%)$ in medium range. The implication for numerical weather and ensemble prediction is that accuracy of diabatic heating initialization affects forecast quality on different circulation scales in different phases of the MJO. 
a) portion of Kelvin variance in IG variance

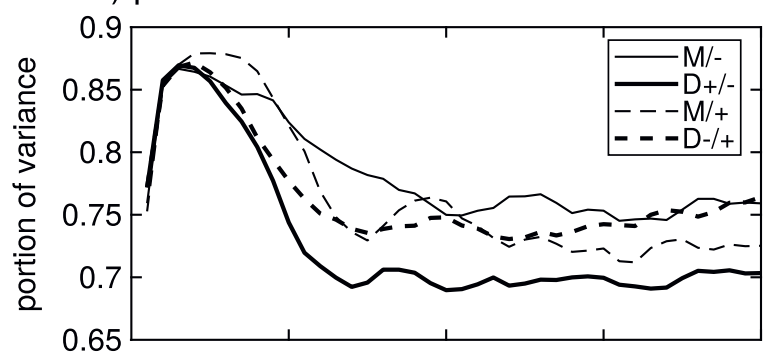

b) portion of $n=1$ in Rossby variance

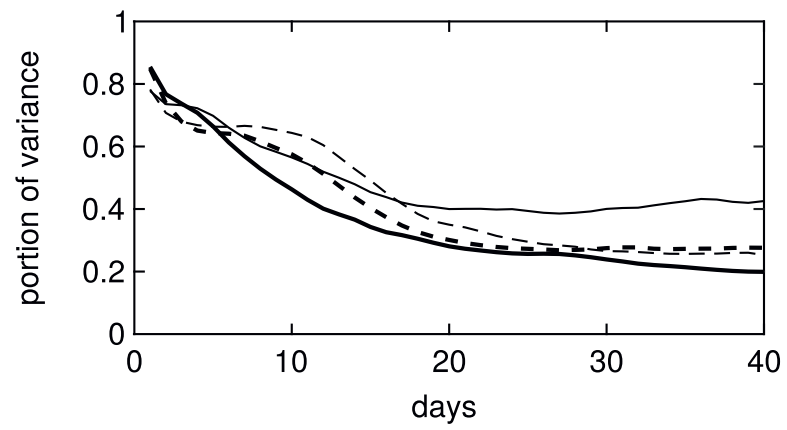

FIG. 9. Evolution of the (a) Kelvin wave variance portion in the IG variance and (b) the $n=1$ Rossby mode variance in the total Rossby variance.

3) The short-term response is projecting predominantly on unbalanced (IG) modes. Day 3 response to perturbations with heating is characterized by about $60 \%$ of variance in IG modes. The majority of it belongs to the Kelvin wave, about $85 \%$ of IG and about $55 \%$ of the total response variance. In the medium (day 14) range, the Kelvin wave variance is at $10 \%$ of the total and $70 \%-80 \%$ of the IG variance. The $n=1$ Rossby mode is the main contributor to the Rossby wave variance at all time scales. However, its variance drops from about $80 \%$ of the Rossby variance and around $30 \%$ of the total variance found on day 3 to $40 \%-50 \%$ of the Rossby and $30 \%-45 \%$ of the total variance on day 14. In other words, other Rossby modes contribute over $50 \%$ of the Rossby wave response to MJO heating. A superposition of the Rossby modes with different $n$ leads to the physical space signal resembling the $n=1$ Rossby mode, especially in the Northern Hemisphere although the cumulative contribution of the $n>1$ Rossby modes in the same zonal wavenumber may exceed that of the $n=1$ Rossby mode.

Acknowledgments. The authors are grateful to the comments of anonymous reviewers that led to paper improvements. We thank our colleagues Martin Horvat,
Blaž Jesenko, Matic Šavli, and Žiga Zaplotnik for helpful discussions during this study. Research of K. Kosovelj and N. Žagar was funded by the European Research Council under the EU's Seventh Framework Programme (FP/2007-2013)/ERC Grant Agreement 280153. N. Žagar is partly funded by the Slovenian Research Agency, Program P1-0188, and Project J1-9431.

\section{REFERENCES}

Adames, A. F., and J. M. Wallace, 2014: Three-dimensional structure and evolution of the MJO and its relation to the mean flow. J. Atmos. Sci., 71, 2007-2026, https://doi.org/ 10.1175/JAS-D-13-0254.1.

Boyd, J. P., and C. Zhou, 2008: Uniform asymptotics for the linear Kelvin wave in spherical geometry. J. Atmos. Sci., 65, 655-660, https://doi.org/10.1175/2007JAS2356.1.

Cassou, C., 2008: Intraseasonal interaction between the MaddenJulian oscillation and the North Atlantic Oscillation. Nature, 455, 523-527, https://doi.org/10.1038/nature07286.

Garcia, R. R., and M. L. Salby, 1987: Transient response to localized episodic heating in the tropics. Part II: Far-field behavior. J. Atmos. Sci., 44, 499-530, https://doi.org/10.1175/15200469(1987)044<0499:TRTLEH > 2.0.CO;2.

Gill, A. E., 1980: Some simple solutions for heat-induced tropical circulation. Quart. J. Roy. Meteor. Soc., 106, 447-462, https:// doi.org/10.1002/qj.49710644905.

Hoskins, B. J., and D. J. Karoly, 1981: The steady linear response of a spherical atmosphere to thermal and orographic forcing. J. Atmos. Sci., 38, 1179-1196, https://doi.org/10.1175/15200469(1981)038<1179:TSLROA > 2.0.CO;2.

Jin, F., and B. J. Hoskins, 1995: The direct response to tropical heating in a baroclinic atmosphere. J. Atmos. Sci., 52, 307-319, https://doi.org/10.1175/1520-0469(1995)052<0307:TDRTTH> 2.0.CO;2.

Kasahara, A., 1976: Normal modes of ultralong waves in the atmosphere. Mon. Wea. Rev., 104, 669-690, https://doi.org/ 10.1175/1520-0493(1976)104<0669:NMOUWI>2.0.CO;2.

_ 1984: The linear response of a stratified global atmosphere to tropical thermal forcing. J. Atmos. Sci., 41, 2217-2237, https:// doi.org/10.1175/1520-0469(1984)041<2217:TLROAS > 2.0.CO;2.

_ , and K. Puri, 1981: Spectral representation of threedimensional global data by expansion in normal mode functions. Mon. Wea. Rev., 109, 37-51, https://doi.org/10.1175/ 1520-0493(1981)109<0037:SROTDG >2.0.CO;2.

_ _ and P. L. da Silva Dias, 1986: Response of planetary waves to stationary tropical heating in a global atmosphere with meridional and vertical shear. J. Atmos. Sci., 43, 1893-1911, https://doi.org/10.1175/1520-0469(1986)043<1893:ROPWTS> 2.0.CO;2.

Kucharski, F., F. Molteni, and A. Bracco, 2006: Decadal interactions between the western tropical Pacific and the North Atlantic Oscillation. Climate Dyn., 26, 79-91, https://doi.org/ 10.1007/s00382-005-0085-5.

,-- , M. P. King, R. Farneti, I.-S. Kang, and L. Feudale, 2013: On the need of intermediate complexity general circulation models: A "SPEEDY" example. Bull. Amer. Meteor. Soc., 94, 25-30, https://doi.org/10.1175/BAMS-D-11-00238.1.

Lee, S. K., C. Wang, and B. E. Mapes, 2009: A simple atmospheric model of the local and teleconnection responses to tropical heating anomalies. J. Climate, 22, 272-284, https://doi.org/ 10.1175/2008JCLI2303.1. 
Lin, H., and G. Brunet, 2018: Extratropical response to the MJO: Nonlinearity and sensitivity to initial state. J. Atmos. Sci., 75, 219-234, https://doi.org/10.1175/JAS-D-17-0189.1.

— - - - , and J. Derome, 2009: An observed connection between the North Atlantic Oscillation and the Madden-Julian oscillation. J. Climate, 22, 364-380, https://doi.org/10.1175/ 2008JCLI2515.1.

-,- , and R. Mo, 2010: Impact of the Madden-Julian oscillation on wintertime precipitation in Canada. Mon. Wea. Rev., 138, 3822-3839, https://doi.org/10.1175/2010MWR3363.1.

Madden, R. A., and P. R. Julian, 1971: Detection of a 40-50 day oscillation in the zonal wind in the tropical Pacific. J. Atmos. Sci., 28, 702-708, https://doi.org/10.1175/1520-0469(1971) 028<0702:DOADOI $>2.0$.CO;2.

__, and _ 1972: Description of global-scale circulation cells in the tropics with a 40-50 day period. J. Atmos. Sci., 29, 1109-1123, https://doi.org/10.1175/1520-0469(1972)029<1109: DOGSCC $>2.0 . \mathrm{CO} ; 2$.

Molteni, F., 2003: Atmospheric simulations using a GCM with simplified physical parametrizations. I: Model climatology and variability in multi-decadal experiments. Climate Dyn., 20, 175-195, https://doi.org/10.1007/s00382-002-0268-2.

Park, C. K., M. J. Suarez, and S. D. Schubert, 1995: Response of the zonally asymmetric flow to time-dependent tropical heating. J. Atmos. Sci., 52, 3738-3756, https://doi.org/10.1175/15200469(1995)052<3738:ROTZAF>2.0.CO;2.

Phillips, N. A., 1990: Dispersion processes in large-scale weather prediction. World Meteorological Organization Rep. 700, 126 pp.

Poli, P., and Coauthors, 2016: ERA-20C: An atmospheric reanalysis of the twentieth century. J. Climate, 29, 4083-4097, https:// doi.org/10.1175/JCLI-D-15-0556.1.

Sardeshmukh, P. D., and B. J. Hoskins, 1988: The generation of global rotational flow by steady idealized tropical divergence. J. Atmos. Sci., 45, 1228-1251, https://doi.org/10.1175/15200469(1988)045<1228:TGOGRF $>2.0$.CO;2.

Seo, K. H., and S. W. Son, 2012: The global atmospheric circulation response to tropical diabatic heating associated with the
Madden-Julian oscillation during northern winter. J. Atmos. Sci., 69, 79-96, https://doi.org/10.1175/2011JAS3686.1.

— nection pattern in response to the MJO. J. Atmos. Sci., 74, 1767-1781, https://doi.org/10.1175/JAS-D-16-0343.1.

Stechmann, S., and A. J. Majda, 2015: Identifying the skeleton of the Madden-Julian oscillation in observational data. Mon. Wea. Rev., 143, 395-416, https://doi.org/10.1175/MWR-D-14-00169.1.

Ting, M., and P. Sardeshmukh, 1993: Factors determining the extratropical response to equatorial diabatic heating anomalies. J. Atmos. Sci., 50, 907-918, https://doi.org/10.1175/15200469(1993)050<0907:FDTERT>2.0.CO;2.

Webster, P. J., and H. R. Chang, 1998: Atmospheric wave propagation in heterogeneous flow: Basic flow controls on tropical-extratropical interaction and equatorial wave modification. Dyn. Atmos. Oceans, 27, 91-134, https://doi.org/10.1016/S0377-0265(97)00003-1.

Welch, B. L., 1947: The generalization of "Student's" problem when several different population variances are involved. Biometrika, 34, 28-35.

Žagar, N., and C. L. E. Franzke, 2015: Systematic decomposition of the Madden-Julian oscillation into balanced and inertio-gravity components. Geophys. Res. Lett., 42, 6829-6835, https://doi.org/ 10.1002/2015GL065130.

_ tivariate modal analysis of atmospheric predictability with application to the ECMWF ensemble. J. Atmos. Sci., 72, 44234444, https://doi.org/10.1175/JAS-D-15-0061.1.

_- A. Kasahara, K. Terasaki, J. Tribbia, and H. Tanaka, 2015b: Normal-mode function representation of global 3-D data sets: Open-access software for the atmospheric research community. Geosci. Model Dev., 8, 1169-1195, https://doi.org/ 10.5194/gmd-8-1169-2015.

Zhang, C., 2005: Madden-Julian oscillation. Rev. Geophys., 43, RG2003, https://doi.org/10.1029/2004RG000158.

Zhong, W., and J. Nogues-Paegle, 1990: Normal mode projections of GCM response to tropical forcing. Tellus, 42A, 429-443, https://doi.org/10.3402/tellusa.v42i4.11888. 